\title{
Studies of quarkonia and doubly-heavy hadrons at LHCb
}

\section{Zhiyu Xiang ${ }^{* \dagger}$}

University of Chinese Academy of Sciences, No.19(A) Yuquan Road, Beijing, China

E-mail: xiangzhiyu16@mails.ucas.ac.cn

The measurements of quarkonia and doubly-heavy hadrons in $p p$ collisions provide crucial inputs to study both perturbative and non-perturbative quantum chromodynamics. LHCb has a broad physics program of studies of quarkonia and doubly-heavy hadrons. In this proceeding, six recent measurements from the $\mathrm{LHCb}$ collaboration are reported: the $\psi(2 S)$ production cross-sections measurement, the updated measurement of $\eta_{c}(1 S)$ production cross-section, the observation of a new charmonium state $\mathrm{X}(3842)$, the observation of an excited $B_{c}^{+}$state, the precision measurement of $B_{c}^{+}$mass, and end this report with the first search of $\Xi_{b c}^{0}$.

40th International Conference on High Energy physics - ICHEP2020

July 28 - August 6, 2020

Prague, Czech Republic (virtual meeting)

\footnotetext{
${ }^{*}$ Speaker

${ }^{\dagger}$ On behalf of the LHCb collaboration
} 


\section{Introduction}

Heavy-flavour hadrons, containing open or hidden charm and beauty flavour, are among the most important tools for the study of Quantum Chromodynamics (QCD) in high-energy hadronic collisions. The heavy-quark mass acts as a long distance cut-off so that the partonic hardscattering process can be calculated in the framework of perturbative QCD (pQCD) down to low transverse momenta $\left(p_{\mathrm{T}}\right)$. Therefore, the studies of quarkonia and doubly-heavy hadrons provides a crucial testing ground for both perturbative and non-perturbative aspects of QCD calculations. The LHCb detector [1,2], with its high momentum resolution, great particle identification capability and flexible trigger strategy, is perfectly suited to study heavy flavour hadrons features.

\section{Measurement of $\psi(2 S)$ production cross-sections}

In high-energy $p p$ collisions, charmonium states can be produced directly from hard collisions, through the feed-down from excited states, or via weak decays of $b$-hadrons. The first two contributions are referred to as prompt production, while the third component can be separated by exploiting the lifetime of $b$-hadrons. For prompt $J / \psi$ production the feed-down contribution is large. This complicates the comparison between theoretical calculations and experimental results. On the contrary, the feed-down contribution to $\psi(2 S)$ mesons is negligible [3], thus theoretical calculations can be directly compared with measurements.

The cross-sections of $\psi(2 S)$ meson production in $p p$ collisions at centre-of-mass energy of $\sqrt{s}=13 \mathrm{TeV}$ are measured with a data sample collected by the LHCb corresponding to an integrated luminosity of $275 \mathrm{pb}^{-1}$ [4]. The production cross-sections for prompt $\psi(2 S)$ and those from $b$ hadron decays $(\psi(2 S)$-form- $b)$ are determined in the rapidity range $2.0<y<4.5$ and in the transverse momentum range $2<p_{\mathrm{T}}<20 \mathrm{GeV} / c$. The production cross-sections integrated over this kinematic region are

$$
\begin{aligned}
& \sigma(\text { prompt } \psi(2 S), 13 \mathrm{TeV})=1.430 \pm 0.005(\text { stat }) \pm 0.099(\text { syst }) \mu \mathrm{b} \\
& \sigma(\psi(2 S) \text {-from }-b, 13 \mathrm{TeV})=0.426 \pm 0.002(\text { stat }) \pm 0.030(\text { syst }) \mu \mathrm{b} .
\end{aligned}
$$

A new measurement of $\psi(2 S)$ production cross-sections at $\sqrt{s}=7 \mathrm{TeV}$ is also performed using data corresponding to an integrated luminosity of $614 \mathrm{pb}^{-1}$. The integrated production cross-sections in the kinematic range $3.5<p_{\mathrm{T}}<14 \mathrm{GeV} / c$ and $2.0<y<4.5$ are

$$
\begin{aligned}
& \sigma(\text { prompt } \psi(2 S), 7 \mathrm{TeV})=0.471 \pm 0.001 \text { (stat) } \pm 0.025 \text { (syst) } \mu \mathrm{b}, \\
& \sigma(\psi(2 S) \text {-from- } b, 7 \mathrm{TeV})=0.126 \pm 0.001 \text { (stat) } \pm 0.008 \text { (syst) } \mu \mathrm{b} .
\end{aligned}
$$

All results show reasonable agreement with theoretical calculations.

\section{Measurement of the $\eta_{c}(1 S)$ production cross-section}

LHCb measured the prompt $\eta_{c}(1 S)$ production cross-section in $p p$ collisions at $\sqrt{s}=7,8 \mathrm{TeV}$ to be below the predictions based on $J / \psi$ prompt production data, and only marginal consistency was found between measurements and theoretical predictions at next-to-leading-order for both prompt production and production in $b$-hadron decays for the $\eta_{c}(1 S)$ and $J / \psi$ [5]. A study of the $\eta_{c}(1 S)$ 
prompt production at $\sqrt{s}=13 \mathrm{TeV}$ [6] provides a further test for theories predicting the $J / \psi$ and $\eta_{c}(1 S)$ hadroproduction cross-sections and the $J / \psi$ polarisation.

Using a data sample corresponding to an integrated luminosity of $2 \mathrm{fb}^{-1}$, the production of the $\eta_{c}(1 S)$ at $\sqrt{s}=13 \mathrm{TeV}$ is studied in the range of $2.0<y<4.5$ and $6.5<p_{\mathrm{T}}<14.0 \mathrm{GeV} / c$. The cross-section for prompt production of $\eta_{c}(1 S)$ mesons relative to that of the $J / \psi$ meson is measured using the $p \bar{p}$ decay mode and is found to be $\sigma_{\eta_{c}(1 S)} / \sigma_{J / \psi}=1.69 \pm 0.15 \pm 0.10 \pm 0.18$. The quoted uncertainties are, in order, statistical, systematic and due to uncertainties on the branching fractions of the $J / \psi \rightarrow p \bar{p}$ and $\eta_{c}(1 S) \rightarrow p \bar{p}$ decays. The prompt $\eta_{c}(1 S)$ production cross-section is determined to be $\sigma_{\eta_{c}(1 S)}=1.26 \pm 0.11 \pm 0.08 \pm 0.14 \mu \mathrm{b}$, where the last uncertainty includes that on the $J / \psi$ cross-section. The ratio of the branching fractions of $b$-hadron decays to the $\eta_{c}(1 S)$ and $J / \psi$ states is measured to be $\mathcal{B}_{b \rightarrow \eta_{c} X} / \mathcal{B}_{b \rightarrow J / \psi X}=0.48 \pm 0.03 \pm 0.03 \pm 0.05$ where the last uncertainty is due to those on the branching fractions of the $\eta_{c}(1 S)$ and $J / \psi$ decays. The difference between the $\eta_{c}(1 S)$ and $J / \psi$ masses is also determined to be $113.0 \pm 0.7 \pm 0.1 \mathrm{MeV} / c^{2}$, which is the most precise single measurement to date.

\section{Observation of a new charmonium state $\mathrm{X}(3842)$}

Plenty of charmonium states have been observed experimentally and their spectrum is well described by the potential models [7]. But still many of the conventional states remain unobserved. Moreover, starting from the discovery of $\chi_{\mathrm{c} 1}(3872)$ more than twenty exotic states which do not fit into the conventional charmonium spectrum are observed. To distinguish between them, it is necessary to account for all of the predicted hidden-charm states, to make the precise measurements of their parameters and to continue a search for new exotic states.

Using the full statistics collected by $\mathrm{LHCb}$, corresponding to an integrated luminosity of 9 $\mathrm{fb}^{-1}$, the $D^{0} \bar{D}^{0}$ and $D^{+} D^{-}$mass spectra are investigated [8]. The $D^{0}$ and $D^{+}$candidates are reconstructed using $D^{0} \rightarrow K^{-} \pi^{+}$and $D^{+} \rightarrow K^{-} \pi^{+} \pi^{+}$decay modes. The $D \bar{D}$ combination mass spectra are shown in Figure 1 (left). Four peaking structures are clearly seen in the spectra. Two of the peaks correspond to the known $\psi(3770)$ and $\chi_{\mathrm{c} 2}(3930)$ charmonium states. A narrow peak close to the threshold represents partially reconstructed $\chi_{\mathrm{c} 1}(3872) \rightarrow D^{* 0} \bar{D}^{0}$ with subsequent $D^{* 0} \rightarrow D^{0} \gamma$ or $D^{* 0} \rightarrow D^{0} \pi^{0}$ decay with $\gamma$ or $\pi^{0}$ missing. The narrow peak with mass about $3840 \mathrm{MeV} / c^{2}$ is identified with a new charmonium state, referred to as $\mathrm{X}(3842)$.

Simultaneous fits to the $D^{0} \bar{D}^{0}$ and $D^{+} D^{-}$combinations mass distributions are performed in three overlapping mass regions in order to better parametrise the background. Fit result is shown in Figure 1 (right) for one of the mass regions containing X(3842) state only. Measured parameters of the new X(3842) state are

$$
\begin{aligned}
m_{\mathrm{X}(3842)} & =3842.71 \pm 0.16 \text { (stat) } \pm 0.12 \text { (syst) } \mathrm{MeV} / c^{2} \\
\Gamma_{\mathrm{X}(3842)} & =2.79 \pm 0.51 \text { (stat) } \pm 0.35 \text { (syst) } \mathrm{MeV}
\end{aligned}
$$

The mass value and the small natural width of the new state suggest the interpretation as the $\psi_{3}\left(1^{3} \mathrm{D}_{3}\right)$ charmonium state with quantum numbers $\mathrm{J}^{\mathrm{PC}}=3^{--}$[9]. In addition, prompt hadroproduction of the $\chi_{\mathrm{c} 2}(3930)$ and $\psi(3770)$ charmonium states are seen for the first time and their parameters are measured to be 

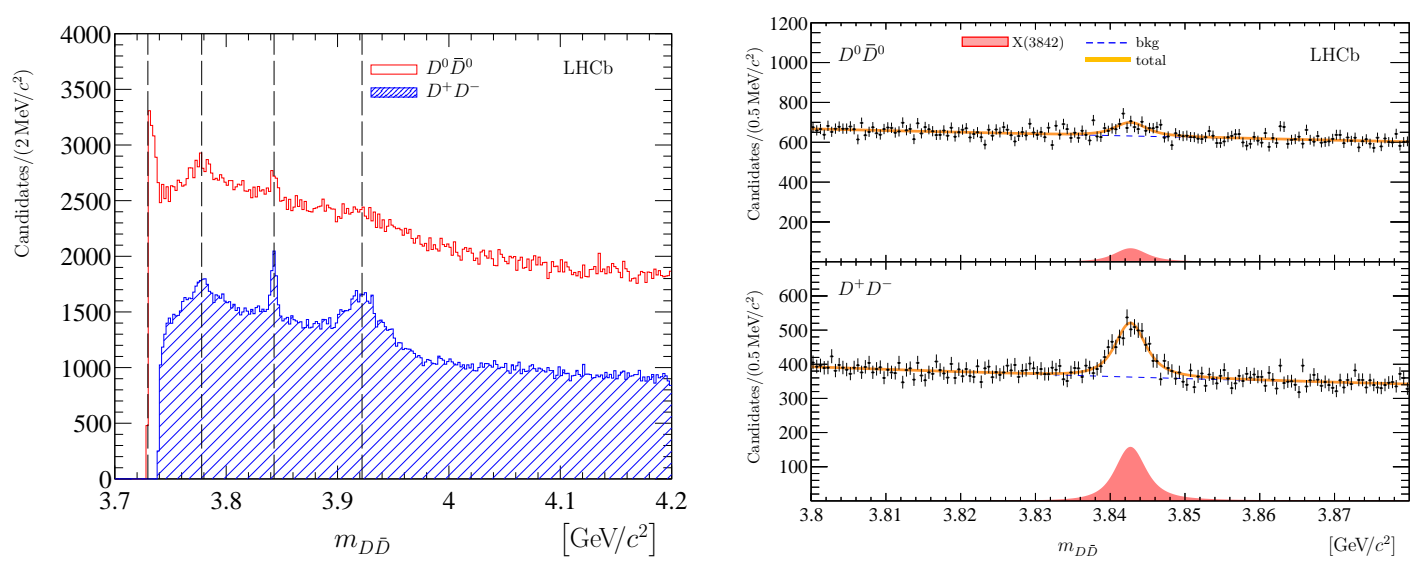

Figure 1: (left) The open red histogram represents $D^{0} \bar{D}^{0}$ combination mass spectra, the blue dashed histogram for $D^{+} D^{-}$combination mass spectra. (right) The (top) $D^{0} \bar{D}^{0}$ and (bottom) $D^{+} D^{-}$combination mass spectra in narrow region. Different components employed in the fit are indicated in the legend.

$$
\begin{aligned}
& m_{\chi_{c 2}(3930)}=3921.9 \pm 0.6(\text { stat }) \pm 0.2 \text { (syst) } \mathrm{MeV} / c^{2}, \\
& \Gamma_{\chi_{c 2}(3930)}=36.6 \pm 1.9 \text { (stat) } \pm 0.9 \text { (syst) } \mathrm{MeV}, \\
& m_{\psi(3770)}=3778.1 \pm 0.7 \text { (stat) } \pm 0.6 \text { (syst) } \mathrm{MeV} / c^{2} .
\end{aligned}
$$

The measured parameters of $\chi_{\mathrm{c} 2}(3930)$ state are in $2 \sigma$ tension with respect to the current world average [3].

\section{Observation of an excited $B_{c}^{+}$state}

The $B_{c}$ meson family has a rich spectrum. There are two $B_{c}(2 S)$ states, $B_{c}\left(2^{1} S_{0}\right)^{+}$and $B_{c}^{*}\left(2^{3} S_{1}\right)^{+}$. The $B_{c}\left(2^{1} S_{0}\right)^{+}$decays to $B_{c}^{+} \pi^{+} \pi^{-}$and $B_{c}^{*}\left(2^{3} S_{1}\right)^{+}$decays to $B_{c}^{*}\left(1^{3} S_{1}\right)^{+}\left(\rightarrow B_{c}^{+} \gamma\right) \pi^{+} \pi^{-}$. The $\gamma$ from the $B_{c}^{*}\left(1^{3} S_{1}\right)^{+}$decay is too soft to reconstruct in LHCb. According to studies in Ref. [10], when $\gamma$ is missing, the reconstructed $B_{c}^{*}\left(2^{3} S_{1}\right)^{+}$mass is shifted down by the mass difference between the $B_{c}^{*}\left(1^{3} S_{1}\right)^{+}$and $B_{c}^{+}$states, and the mass resolution is only slightly degraded.

Using $p p$ collision data corresponding to an integrated luminosity of $8.5 \mathrm{fb}^{-1}$ recorded by the LHCb at $\sqrt{s}=7,8$ and $13 \mathrm{TeV}$, the excited $B_{c}^{*}\left(2^{3} S_{1}\right)^{+}$state in the $B_{c}^{+} \pi^{+} \pi^{-}$invariant-mass spectrum is observed [11], as shown in Figure 2. The $\Delta M \equiv M\left(B_{c}^{+} \pi^{+} \pi^{-}\right)-M\left(B_{c}^{+}\right)$peak with mass about $566 \mathrm{MeV} / c^{2}$ is consistent with expectations of the $B_{c}^{*}\left(2^{3} S_{1}\right)^{+}$. The observed peak has a reconstructed mass of $6841.2 \pm 0.6$ (stat) \pm 0.1 (syst) $\pm 0.8\left(\mathrm{~B}_{\mathrm{c}}^{+}\right) \mathrm{MeV} / c^{2}$, where the last uncertainty is due to the limited knowledge of the $B_{c}^{+}$mass. A second state is seen with a global (local) statistical significance of $2.2 \sigma(3.2 \sigma)$ and a mass of $6872.1 \pm 1.3$ (stat) \pm 0.1 (syst) $\pm 0.8\left(\mathrm{~B}_{\mathrm{c}}^{+}\right) \mathrm{MeV} / c^{2}$, and is consistent with the $B_{c}\left(2{ }^{1} S_{0}\right)^{+}$state. These mass measurements are the most precise to date.

\section{Precision measurement of $B_{c}^{+}$meson mass}

Studies of the $B_{c}$ meson can reveal information on heavy-quark dynamics and improve our understanding of the strong interaction. Due to the presence of two heavy-quarks the mass spectrum of the $B_{c}$ states can be predicted with much better precision than many other hadronic systems. 


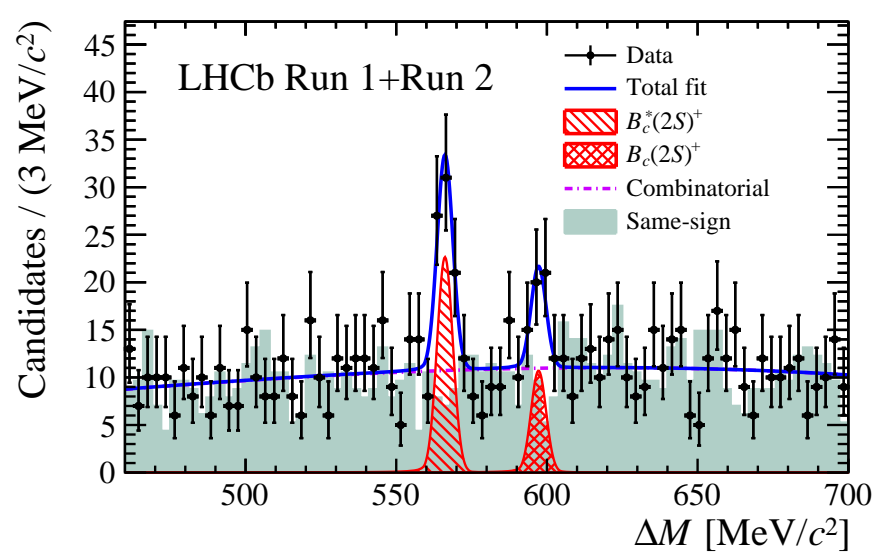

Figure 2: Distribution of $\Delta M=M\left(B_{c}^{+} \pi^{+} \pi^{-}\right)-M\left(B_{c}^{+}\right)$with the fit results overlaid. The same-sign distribution has been normalized to the data in the $B_{c}^{*}\left(2^{3} S_{1}\right)^{+}$sideband region.

The world average of the $B_{c}^{+}$mass has an uncertainty of $0.8 \mathrm{MeV} / c^{2}$ [3], which is the dominant systematic uncertainty in the $B_{c}^{(*)}(2 S)^{+}$mass measurements [11].

A precision measurement of the $B_{c}^{+}$mass is performed using $p p$ collision data collected with LHCb at $\sqrt{s}=7,8$ and $13 \mathrm{TeV}$, corresponding to an integrated luminosity of $9 \mathrm{fb}^{-1}$ [12]. Combining the results of the individual decay channels: $B_{c}^{+} \rightarrow J / \psi \pi^{+}, B_{c}^{+} \rightarrow J / \psi \pi^{+} \pi^{-} \pi^{+}, B_{c}^{+} \rightarrow J / \psi p \bar{p} \pi^{+}$, $B_{c}^{+} \rightarrow J / \psi D_{s}^{+}, B_{c}^{+} \rightarrow J / \psi D^{0} K^{+}$and $B_{c}^{+} \rightarrow B_{s}^{0} \pi^{+}$, the $B_{c}^{+}$meson mass is measured to be $6274.47 \pm 0.27$ (stat) \pm 0.17 (syst) $\mathrm{MeV} / c^{2}$. This is the most precise measurement of the $B_{c}^{+}$ meson mass to date. In addition, the difference between $B_{c}^{+}$and $B_{s}^{0}$ masses is measured to be $907.75 \pm 0.37$ (stat) \pm 0.27 (syst) $\mathrm{MeV} / c^{2}$.

\section{First search for $\Xi_{b c}^{0} \rightarrow D^{0} p K^{-}$}

The baryons with two heavy quarks are of interest for the theoretical consideration, since for their description would combined approach involving the features of the effective theory of heavy quarks (HQET), nonrelativistic QCD (NRQCD) and potential nonrelativistic QCD (pNRQCD) because in this system the interaction of light quark with the heavy quarks is essential as well as the interaction between the heavy quarks.

A first search for the doubly-heavy $\Xi_{b c}^{0}$ baryon using its decay to the $D^{0} p K^{-}$final state is performed using $p p$ collision data at $\sqrt{s}=13 \mathrm{TeV}$ collected by $\mathrm{LHCb}$, corresponding to an integrated luminosity of $5.4 \mathrm{fb}^{-1}$ [13]. No significant signal is found in the invariant mass range from 6.7 to $7.2 \mathrm{GeV} / c^{2}$. Upper limits are set at $95 \%$ credibility level on the ratio of the $\Xi_{b c}^{0}$ production cross-section times its branching fraction to $D^{0} p K^{-}$relative to that of the $\Lambda_{b}^{0} \rightarrow D^{0} p K^{-}$ decay, in the region of $5<p_{\mathrm{T}}<25 \mathrm{GeV} / c$ and $2.0<y<4.5$. Upper limits range from $1.7 \times 10^{-2}$ to $3.0 \times 10^{-1}$ for the considered $\Xi_{b c}^{0}$ lifetime hypotheses, 100 to $500 \mathrm{fs}$.

\section{Summary}

Several recent $\mathrm{LHCb}$ results on quarkonia and doubly-heavy hadrons in $p p$ collisions have been reported. More precise measurement of $\psi(2 S)$ production at $\sqrt{s}=7$ and $13 \mathrm{TeV}$ show reasonable 
agreement with theoretical prediction. First $\eta_{c}(1 S)$ production measurement at $\sqrt{s}=13 \mathrm{TeV}$ is in $2 \sigma$ agrees with prediction by the colour-singlet model [14]. A new charmonium state $\mathrm{X}(3842)$ is observed. The prompt hadroproduction of $\psi(3770)$ and $\chi_{\mathrm{c} 2}(3930)$ states are first observed. Observation of excited $B_{c}^{(*)}(2 S)^{+}$and most precise mass measurement to date. Most precise measurement of $B_{c}^{+}$mass. First search for $\Xi_{b c}^{0} \rightarrow D^{0} p K^{-}$and upper limit set. More results of quarkonia and doubly-heavy hadrons at $\mathrm{LHCb}$ are expected in the near future.

\section{References}

[1] LHCb Collaboration, A. Alves et al., The LHCb Detector at the LHC, JINST 3 (2008), S08005 doi:10.1088/1748-0221/3/08/S08005.

[2] LHCb Collaboration, R. Aaij et al., The LHCb Detector Performance, Int. J. Mod. Phys. A 30 (2015) no.07, 1530022 doi:10.1142/S0217751X15300227, arXiv:1412.6352.

[3] Particle Data Group, M. Tanabashi et al., Review of particle physics, Phys. Rev. D98 (2018) 030001.

[4] LHCb Collaboration, R. Aaij et al., Measurement of $\psi(2 S)$ production cross-sections in protonproton collisions at $\sqrt{s}=13 \mathrm{TeV}$, Eur. Phys. J. C 80 (2020) no. 3, 185, arXiv:1908.03099.

[5] LHCb collaboration, R. Aaij et al., Measurement of the $\eta_{c}(1 S)$ production crosssection in $p p$ collisions via the decay $\eta_{c}(1 S) \rightarrow p \bar{p}$, Eur. Phys. J. C75 (2015) 311, arXiv:1409.3612.

[6] LHCb Collaboration, R. Aaij et al., Measurement of the $\eta_{c}(1 S)$ production cross-section in $p p$ collisions at $\sqrt{s}=13$ TeV, Eur. Phys. J. C80 no. 3, (2020) 191, arXiv:1911.03326.

[7] E. Eichten, K. Gottfried, T. Kinoshita, K.D. Lane, T.M. Yan, Charmonium: The model, Phys. Rev. D17, 3090 (1978), [Erratum: Phys. Rev.D21,313(1980)].

[8] LHCb collaboration, R. Aaij et al., Near-threshold $D \bar{D}$ spectroscopy and observation of a new charmonium state, JHEP 07 (2019) 035, arXiv:1903.12240.

[9] T. Barnes, S. Godfrey, E.S. Swanson, Higher charmonia, Phys. Rev. D 72, 054026 (2005).

[10] Y.-N. Gao et al., Experimental prospects of the $B_{c}$ studies of the LHCb experiment, Chin. Phys. Lett. 27 (2010) 061302.

[11] LHCb collaboration, R. Aaij et al., Observation of an excited $B_{c}^{+}$state, Phys. Rev. Lett. 122, 232001 (2019), arXiv:1904.00081.

[12] LHCb collaboration, R. Aaij et al., Precision measurement of the $B_{c}^{+}$meson mass, JHEP 07 (2020) 123, arXiv:2004.08163.

[13] R. Aaij et al. [LHCb], Search for the doubly-heavy $\Xi_{b c}^{0}$ baryon via decays to $D^{0} p K^{-}$, JHEP 11 (2020) 095, arXiv:2009.02481.

[14] Y. Feng et al., Phenomenological NLO analysis of $\eta_{c}$ production at the LHC in the collider and fixed-target modes, Nucl. Phys. B (2019) 114662, arXiv:1901.09766. 